\title{
Correction to: Transcriptional Regulators as Targets for Alcohol Pharmacotherapies
}

Antonia M. Savarese and Amy W. Lasek

\section{Correction to: \\ (C) Springer International Publishing AG 2018 Handbook of Experimental Pharmacology, https://doi.org/10.1007/164_2018_101}

Figure 1 was published incorrectly in this chapter. The original chapter was corrected. The correct Figure 1 is 


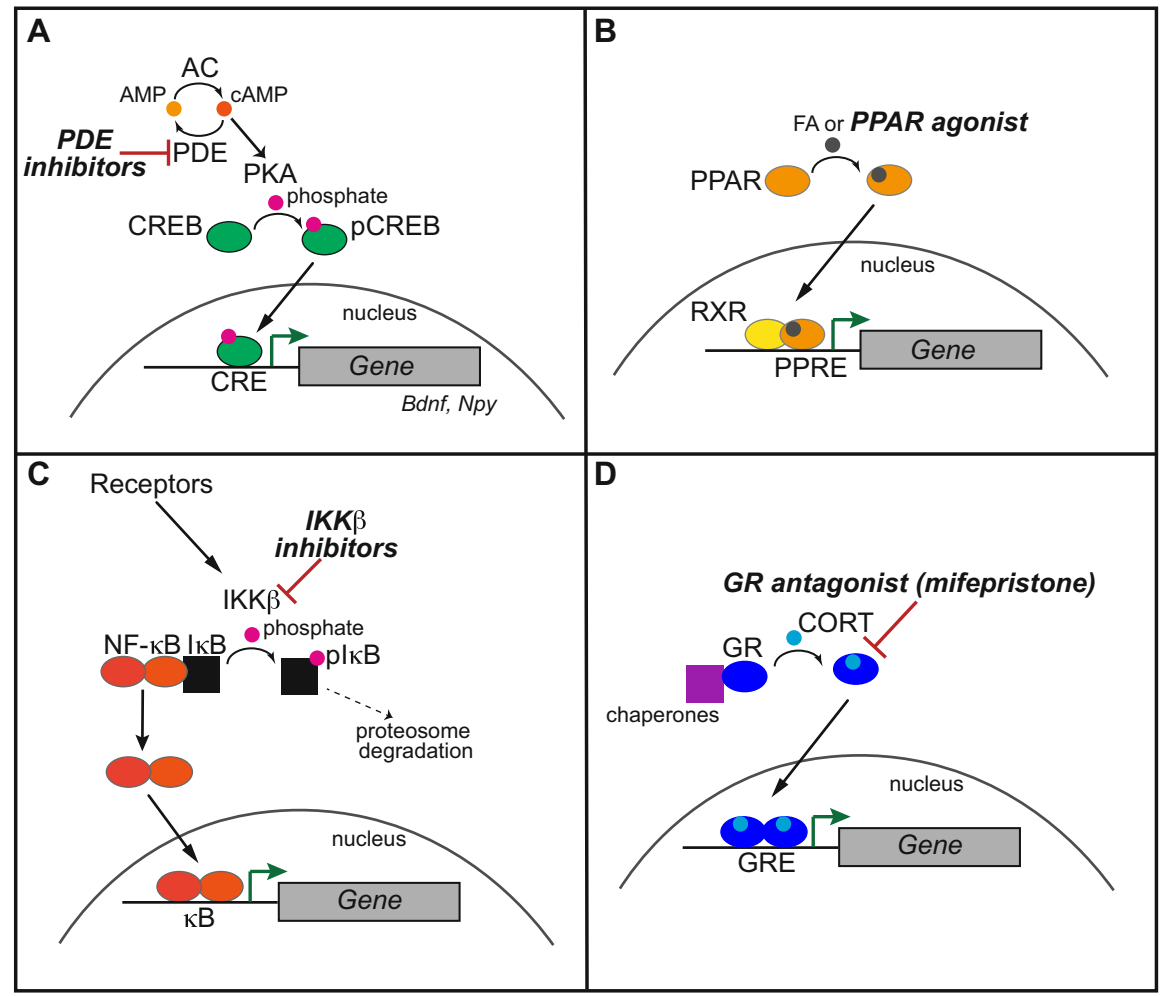

Fig. 1 Simplified diagram of transcriptional pathways and targets for intervention for alcohol use disorder (AUD) treatment. (a) The cAMP-responsive element binding protein (CREB) pathway. Adenylyl cyclase (AC) produces cAMP from AMP, activating protein kinase A (PKA). CREB is phosphorylated (pCREB) by several kinases, one of which is PKA. Once phosphorylated, CREB translocates to the nucleus and binds to cAMP-responsive elements (CRE) in the DNA to activate transcription of genes associated with AUD such as Bdnf and Npy. One method to activate CREB is to use compounds that inhibit the phosphodiesterases (PDEs) that hydrolyze cAMP, thus increasing cAMP levels and activating PKA. PDE inhibitors reduce alcohol consumption in animal models of AUD. (b) The peroxisome proliferator-activated receptor (PPAR) signaling pathway. PPARs are activated by their endogenous ligands, fatty acids (FA), or by synthetic agonists such as the thiazolidinediones and fibrates. Upon ligand binding, PPARs translocate to the nucleus and interact with retinoid X receptor (RXR) at peroxisome proliferator response elements (PPREs) to regulate gene transcription. PPAR agonists reduce alcohol consumption in animal models of AUD. (c) The nuclear factor

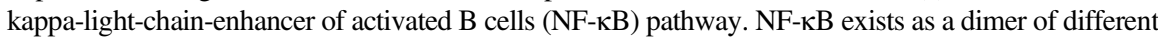
subunits and is complexed with an inhibitory molecule, inhibitor $\kappa \mathrm{B}(\mathrm{I \kappa B})$ in the cytosol. Activation of various receptors leads to activation of IкB kinase (IKK $\beta)$ and phosphorylation of IкB. This event targets IкB for degradation, releasing NF- $\kappa \mathrm{B}$ for translocation to the nucleus to regulate gene expression at $\kappa B$ elements. IKK $\beta$ inhibitors reduce alcohol consumption in mice. (d) Glucocorticoid receptor (GR) pathway. GR is held in the cytosol by chaperone proteins. Once bound to its ligand, cortisol (in humans/nonhuman primates) or corticosterone (in rodents) (CORT), GR translocates to the nucleus and binds to glucocorticoid response elements (GREs) to regulate gene transcription. The GR antagonist mifepristone has shown efficacy in reducing alcohol consumption in rodents and humans 